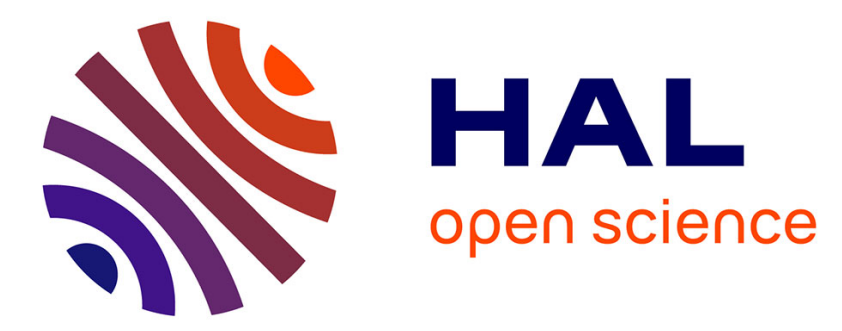

\title{
Effets des radiofréquences sur le système nerveux central chez l'homme: EEG, sommeil, cognition, vascularisation
}

Rania Ghosn, Anne-Sophie Villegier, Brahim Selmaoui, György Thuróczy, René de Seze

\section{- To cite this version:}

Rania Ghosn, Anne-Sophie Villegier, Brahim Selmaoui, György Thuróczy, René de Seze. Effets des radiofréquences sur le système nerveux central chez l'homme: EEG, sommeil, cognition, vascularisation. Comptes Rendus. Physique, 2013, 14 (5), pp.395-401. 10.1016/j.crhy.2013.02.004 . ineris-00961807

HAL Id: ineris-00961807

https://hal-ineris.archives-ouvertes.fr/ineris-00961807

Submitted on 20 Mar 2014

HAL is a multi-disciplinary open access archive for the deposit and dissemination of scientific research documents, whether they are published or not. The documents may come from teaching and research institutions in France or abroad, or from public or private research centers.
L'archive ouverte pluridisciplinaire HAL, est destinée au dépôt et à la diffusion de documents scientifiques de niveau recherche, publiés ou non, émanant des établissements d'enseignement et de recherche français ou étrangers, des laboratoires publics ou privés. 


\title{
Effets des RF sur le système nerveux central chez l'homme : EEG, sommeil, cognition, vascularisation
}

\author{
Effects of RF on the central nervous system in human: \\ EEG, sleep, cognition, vascularisation
}

\author{
Rania Ghosn, Anne-Sophie Villégier, Brahim Selmaoui, Georges Thuróczy et René de Seze (corresponding author) \\ Unité Mixte PériTox EA 4285 - UMI 01 - 1) INERIS Parc ALATA BP2, 60550 Verneuil-en-Halatte, France 2) UPJV \\ UFR de Médecine, 3, rue des Louvels, 80036 Amiens, France, \\ Tel : +33.3.44.55.61.67; Fax : +33.3.44.55.68.00; e-mail : rene.de-seze@ineris.fr
}

Mots-clefs: RF, système nerveux central, EEG, sommeil, cognition, vascularisation

Key words: RF, central nervous system, EEG, sleep, cognition, vascularisation

\begin{abstract}
Most of human studies with radiofrequency electromagnetic fields (RF) looked at of mobile phones-related exposures, usually at the level of the head, on some physiological functions including sleep, brain electrical activity (EEG), cognitive processes, brain vascularisation, and more generally on the cardiovascular and endocrine systems. They were frequently performed on healthy adults. Effects on amplitude of EEG alpha waves, mainly during sleep, look consistent. It would however be important to define more precisely whether and how the absence of electromagnetic disturbance between RF exposure and recording systems is checked. No consensus arises about cognitive effects. Some effects on cerebral vascularisation need complementary work.
\end{abstract}

\begin{abstract}
Résumé
La plupart des études cliniques avec des champs radiofréquences (RF) ont porté sur les effets d'expositions caractéristiques des téléphones portables, habituellement au niveau de la tête, sur un certain nombre de fonctions physiologiques comprenant le sommeil, l'activité électrique du cerveau (EEG), la cognition, la vascularisation cérébrale et plus généralement les systèmes cardiovasculaire et endocrinien. La majorité des études a été effectuée chez des adultes en bonne santé. L'effet décrit sur l'amplitude des ondes alpha de l'EEG, notamment pendant le sommeil, semble reproductible. Il semble cependant important de préciser si et comment l'absence d'interférence entre l'exposition RF et l'enregistrement est vérifiée. Il ne se dégage pas de consensus sur des effets cognitifs. Quelques effets décrits sur la vascularisation cérébrale nécessitent des études complémentaires.
\end{abstract}

\section{Introduction}

De nombreuses études cliniques avec des champs RF ont étudié les effets d'expositions caractéristiques des téléphones portables, habituellement au niveau de la tête, sur un certain nombre de paramètres physiologiques comprenant le sommeil, l'activité électrique du cerveau, la cognition, et la vascularisation cérébrale. Cet article fait une synthèse des études effectuées et essaie d'en dégager les principaux résultats.

\section{2. Études sur l'EEG et le sommeil}

L'électroencéphalogramme (EEG) trace l'activité électrique des cellules nerveuses du cortex sur le cuir chevelu. L'EEG se compose de " bandes " distinctes définies par la fréquence des ondes. Les bandes à basse fréquence (8 à $12 \mathrm{~Hz})$, appelées bandes alpha, s'observent mieux dans la zone pariéto-occipitale. Celles à plus haute fréquence, appelées bandes bêta $(12$ à $30 \mathrm{~Hz})$, sont habituellement proéminentes dans les zones frontale et centrale. Le rythme alpha s'observe mieux lorsque le sujet est éveillé mais calme et qu'il a les yeux fermés.

Dans une revue, Cook et al. [1] notent que l'EEG et autres techniques similaires peuvent s'utiliser plus facilement sur des sujets volontaires que les méthodes d'imagerie cérébrale, car elles ne produisent aucun rayonnement ionisant ni aucun champ magnétique élevé. Il peut toutefois exister des interférences provenant des champs d'extrêmement basses fréquences (ou Extremely Low Frequencies en anglais (ELF) et radiofréquences. 


\subsection{EEG d'éveil}

Roschke et Mann [2]n'ont révélé aucun changement chez des volontaires de sexe masculin exposés à $900 \mathrm{MHz}$, et Hietanen et al. [3] n'ont observé aucun effet sur les EEG suite à une exposition à des téléphones mobiles utilisant des fréquences de 900 et de $1800 \mathrm{MHz}$. Utilisant un EEG télémétrique, Kramarenko et al. [4] ont constaté qu'après une exposition de 20 à 40 secondes à un signal téléphonique de $900 \mathrm{MHz}$, les sujets présentaient une activité à ondes lentes dans les zones frontales et temporales controlatérales, qui durait une seconde et se répétait toutes les 15 à 20 secondes. Les ondes lentes ont progressivement disparu dans les dix minutes qui ont suivi la fin de l'exposition. Hinrikus et al. [5] ont observé des changements dans le rythme alpha de certains sujets, sans pour autant noter de changement statistiquement significatif entre l'exposition réelle et l'exposition factice (appelée Sham). Vecchio et al. [6] ont mesuré la puissance spectrale de l'EEG chez des volontaires masculins sains, qui ont été exposés au signal d'un téléphone GSM situé sur le côté gauche de la tête, contrebalancée par un téléphone non-opérationnel sur le côté droit. L'exposition a modifié la connectivité ${ }^{1}$ entre les deux hémisphères du cerveau dans certaines parties de la bande alpha (8-12 Hz). Dans les zones frontales du cerveau la connectivité a diminué, tandis qu'elle a augmenté dans les régions temporales.

La plupart des études montrent une augmentation de la puissance des bandes alpha de l'EEG des sujets éveillés au repos. Reiser et al. [7] ont observé une augmentation de cette puissance dans les bandes de fréquences alpha2, beta1 et beta2 dans la région occipitale pendant et après l'exposition aux champs RF à $900 \mathrm{MHz}$, mais d'autres chercheurs ont fait valoir que de tels changements peuvent être provoqués par une modification du niveau d'attention. Huber et al. [8] ont étudié l'effet du signal GSM $900 \mathrm{MHz}$ modulé par impulsions et l'effet du signal $900 \mathrm{MHz}$ continu sur l'EEG pendant l'éveil. Le côté gauche de la tête de chaque volontaire a été exposé à ces deux types de signaux pendant 30 min avant endormissement, sur 3 soirées distinctes à des intervalles hebdomadaires. Ils ont observé une augmentation de puissance dans la gamme alpha pendant l'exposition à un signal modulé par impulsions, mais pas au signal d'onde continu. Regel et al. [9] ont obtenu des résultats similaires, 30 minutes après une exposition à des ondes modulées par impulsion, mais n'ont également observé aucun changement durant une exposition à des ondes continues. Curcio et al. [10] ont enregistré l'EEG chez la moitié des sujets après la fin de l'exposition et durant les 7 dernières min d'exposition dans l'autre moitié. Une légère augmentation dans les fréquences 9 et $10 \mathrm{~Hz}$ de la bande alpha a été mentionnée. Cette augmentation était plus prononcée durant l'exposition qu'après la fin de l'exposition. Selon Cook et al. [11], l'activité des bandes alpha était significativement plus élevée dans la région occipitale après 15 min d'exposition au champ électromagnétique pulsé par rapport à l'exposition fictive. De même, Hinrikus et al. [12] ont trouvé une augmentation dans la puissance des bandes alpha $(8-13 \mathrm{~Hz})$ et beta $(15-20$ et 22-38 Hz) pendant la moitié de la première minute de chaque exposition à un signal radiofréquence de $450 \mathrm{MHz}$ avec modulation d'impulsion à 14 et $21 \mathrm{~Hz}$. La variabilité entre les sujets était élevée et les moyennes rapportées ont été fortement influencées par les valeurs de quelques sujets. Croft et al. [13] ont réalisé une étude avec un large nombre de volontaires adultes $(\mathrm{n}=120)$ exposés à un téléphone GSM $875 \mathrm{MHz}$ dans une étude en double aveugle et ils ont évalué l'EEG dans les premières et dernières $10 \mathrm{~min}$ des $30 \mathrm{~min}$ d'exposition. Une augmentation globale de la puissance des bandes alpha lors de l'exposition a été confirmée (par rapport au contrôle Sham), avec effet supérieur du côté homolatéral par rapport au côté controlatéral au niveau des régions postérieures. Il n'a pas été observé de changement dans l'activité des bandes alpha après l'arrêt de l'exposition. Ces résultats confirment ceux des études précédentes qui ont rapporté un effet de l'exposition RF sur la puissance de l'EEG dans la bande alpha. Comme dans les études antérieures, Croft et al. [14] ont trouvé une augmentation dans cette bande alpha chez les jeunes adultes suite à l'exposition $2 \mathrm{G}$ par rapport à l'exposition Sham, cependant, aucun effet n'a été observé dans les groupes d'adolescents ou de personnes âgées. D'autre part, aucun effet de l'exposition 3G n'a été trouvé dans les trois groupes d'âges déjà cités.

Huber et al. [15] ont observé une diminution de la puissance de l'EEG de repos dans la gamme de fréquence entre 10,5 et $11 \mathrm{~Hz}$ après l'exposition des volontaires sains pendant $30 \mathrm{~min}$ à un signal GSM $900 \mathrm{MHz}$, immédiatement avant l'endormissement. Quant à Croft et al. [16], ils ont constaté une diminution de l'activité dans l'hémisphère droit, dans la bande de fréquences de 1-4 Hz, et une augmentation tributaire de la durée d'exposition dans la bande des fréquences 8 $12 \mathrm{~Hz}$, dans les zones postérieures à la ligne médiane. Dans cette étude, la durée d'exposition était 20 min et le téléphone $900 \mathrm{MHz}$ a été maintenu en position radiale à $5 \mathrm{~cm}$ du cuir chevelu entre $\mathrm{Oz}$ et $\mathrm{Pz}$ à l'aide d'un support métallique externe.

\subsection{EEG de sommeil}

Le tracé normal de l'EEG varie dans les deux stades principaux de sommeil. Ainsi, les ondes bêta sont plus fréquentes durant le sommeil paradoxal (mouvements oculaires rapides), que l'on associe au stade de traitement de l'information. Le stade de sommeil lent est quant à lui associé aux ondes delta et thêta. Les deux stades durent environ 90 minutes et se répètent environ cinq fois par nuit.

Quelques études ont examiné les effets du rayonnement RF sur des sujets endormis. Wagner et al. [17] n'ont pas réussi à reproduire les résultats antérieurs de son groupe [18] faisant état d'un effet de suppression des mouvements oculaires rapides (REM) avec réduction de la durée et du pourcentage de REM et d'une augmentation de la puissance spectrale de l'EEG durant le sommeil REM chez des hommes volontaires sains exposés à des champs électromagnétiques de 900 MHz. Borbely et al. [19] ont observé un nombre réduit d'épisodes d'éveil après l'endormissement et des changements

\footnotetext{
${ }^{1}$ Cohérence du spectre entre les paires d'électrodes droites et gauches (F3-F4, C3-C4, P3-P4)
} 
dans les spectres de puissance EEG lors du premier épisode de sommeil lent de la nuit qui se traduisent par une augmentation significative de la puissance de l'EEG dans les fréquences entre 11 et 11,5 Hz. Aucun effet sur la latence et l'état de sommeil n'a été observé. Selon Huber et al. [15] une exposition de 30 minutes à un champ RF avant le sommeil modifiait l'EEG enregistré pendant la période de sommeil suivante en augmentant la puissance spectrale dans les bandes alpha et bêta dans la première phase de sommeil. Toutefois, aucune différence n'a été relevée dans la latence du sommeil, dans les stades de sommeil ni dans l'éveil des sujets endormis. Les mêmes auteurs (Huber et al. [8]) ont trouvé des résultats similaires dans une étude menée en 2002, bien que les changements n'aient alors été observés qu'avec un signal modulé par impulsions.

Lebedeva et al. [20] ont découvert une augmentation de la densité de puissance alpha et des modifications de la structure du sommeil, mais fournissent peu de détails sur l'exposition RF utilisée dans leur expérience. Loughran et al. [21] ont constaté une diminution de la latence du sommeil paradoxal. Ces auteurs notent également une augmentation de la densité spectrale de puissance dans la gamme alpha, durant la période initiale du sommeil suivant l'exposition. D'autres groupes ont aussi observé ce résultat (Borbely et al. [22] et Huber et al. [15]).

Hung et al. [23] ont étudié l'effet de signaux GSM $900 \mathrm{MHz}$ avec différentes modulations d'impulsions ELF sur l'endormissement et l'architecture du sommeil. Ils ont exposé les sujets à un téléphone mobile en mode "conversation", « écoute », « veille » et "exposition sham". Ils ont constaté que, après l'exposition en mode "conversation", il y avait une augmentation dans la latence du sommeil par rapport au mode "écoute" et "exposition sham". Le Débit d'absorption spécifique (DAS) était le plus élevé en mode "conversation" et aux modulations d'impulsions 8 et 217 Hz.

Les études sur les effets des ondes RF de la téléphonie mobile sur l'EEG d'éveil paraissent trop fluctuantes et non concluantes. Cependant, plusieurs études montrent un effet de l'exposition principalement sur les bandes alpha du spectre EEG. Les études de l'EEG de sommeil paraissent avoir des résultats plus robustes que les études EEG d'éveil. Les deux observations majeures sont une augmentation dans la densité spectrale de puissance dans la gamme alpha durant la période initiale du sommeil suivant l'exposition et aucun changement dans la latence du sommeil, ni dans les stades de sommeil, ni dans l'éveil après endormissement. Dans plusieurs études, les informations sur la maîtrise de l'interférence entre le champ électromagnétique et les électrodes d'EEG n'ont pas été fournies. Une telle interférence peut aboutir à des résultats erronés. D’où la nécessité dans les nouvelles études de préciser la méthodologie de contrôle d'éventuelles interférences qui pourraient aboutir à des résultats douteux.

\section{Effets sur les fonctions cognitives}

Une large gamme de tests comportementaux permet d'examiner les fonctions cognitives et d'attention chez l'Homme. Ces tests incluent des réponses à un stimulus simple (test du temps de réaction (TR) simple) et des tests plus complexes faisant intervenir un traitement plus élaboré de l'information et la présentation de plusieurs stimuli et des choix (tests d'attention, test «n-back», ...). Un certain nombre d'études ont examiné les effets des expositions aux champs électromagnétiques du téléphone portable sur les fonctions cognitives par l'utilisation de ces tests (pour revue : Kwon et Hämäläinen [24]).

Plusieurs arguments expérimentaux indiquent une tendance à un effet facilitateur sur la cognition des expositions aux champs électromagnétiques des téléphones portables. En effet, les premières études rapportaient une amélioration des performances sous exposition dans des tâches d'attention et de mémoire de travail [25-28]. Preece et al. [25] ont rapporté une réduction dépendante de la puissance du TR dans une tâche de choix $(1 \mathrm{~W}<0,125 \mathrm{~W}<\operatorname{sham~} 0 \mathrm{~W})$.

Sur les mêmes tâches d'attention et de mémoire adaptées pour les enfants, Preece et al. [29] ont testé si des effets pouvaient être observés lors d'émissions à 0,25 et $0,025 \mathrm{~W}$. Leurs résultats ont montré une tendance à l'amélioration des performances, notamment une réduction dans le test de TR simple $(\mathrm{P}<0,02)$, pour la plus forte puissance.

Koivisto et al. [30] [26, 27] ont rapporté une réduction du TR dans des tâches de mémoire de travail (3-back) et des mesures du TR (simple, soustraction, vigilance). Curcio et al. [31] ont montré une réduction des TR simples $(\mathrm{P}<0,004)$ et de choix $(\mathrm{P}<0,007)$ pour six variables. Keetley et al. [32] ont rapporté une amélioration du temps de réactivité dans la tâche de «trail-making ». Une méta-analyse conduite sur 10 études [24] concernant les effets des signaux GSM sur les fonctions d'attention et de mémoire révélait une réduction du TR dans les tâches de soustraction $(\Delta=-0,09,95 \%$ CI: $-0,16$ to $-0,02)$, du RT dans le test « 0 -back target » $(\Delta=-0,04,95 \%$ CI: $-0,06$ à $-0,02)$. Wiholm et al. [33] ont rapporté que les performances de navigation virtuelle chez un groupe de sujets hypersensibles étaient améliorées à la suite d'une exposition réelle.

Cependant, un certain nombre d'éléments limitants dans les expérimentations ou l'interprétation des résultats ne permettent pas de conclure à un effet facilitateur des champs électromagnétiques RF sur la cognition. Ainsi, dans le premier cas, les études testaient un large nombre de variables obtenues à partir d'une série de tâches sans y apporter les corrections appropriées en lien avec les comparaisons multiples [25-28]. De plus, ces premiers résultats n'ont pas toujours été confirmés, en particulier au sein des mêmes groupes de recherche. Certains résultats, comme la réduction puissance-dépendante du TR dans une tâche de choix étaient les seuls significatifs $(\mathrm{P}<0,003)$ parmi 15 variables testées obtenues à partir de dix tâches d'attention et de mémoire [25].

Dans la plupart des études, malgré des tendances à l'amélioration des performances, aucune des variables n'atteint la significativité statistique après les corrections de Bonferroni (22 tests) [29]. De même, malgré des tendances, les TR 
dans les tâches simples $(\mathrm{P}<0,026)$ et de soustraction $(\mathrm{P}<0,044)$ n'ont pas atteint la significativité statistique après correction en lien avec les tests multiples (14 tests) [26, 27]. Finalement, une diminution des performances en lien avec l'exposition sham ayant probablement eu lieu par hasard peut expliquer les effets apparents des champs électromagnétiques, en l'absence d'autres différences parmi les groupes hypersensibles (exposition réelle) et contrôles (exposition réelle, exposition sham) [33].

En réponse à ces faiblesses dans les protocoles et les analyses, des ajustements ont été proposés. Les études de confirmation avec amélioration du protocole de l'étude (taille d'échantillon plus importante, double aveugle, etc.) n'ont pas montré d'effet significatif dans les mesures des TR [34] ou de mémoire de travail [35, 36]. Des études complémentaires incluant des conditions supplémentaires d'exposition [37] et les enfants [38] n'ont pas non plus montré d'effet significatif. Russo et al. [39] et Cinel et al. [40, 41] ont testé l'effet facilitateur de la cognition sur les fonctions d'attention [27], de mémoire [26], sur un large échantillon de 168 sujets pour augmenter le pouvoir statistique, mais aucune des études n'a montré d'effet significatif.

Cependant, des arguments expérimentaux complémentaires ont permis de rejeter l'hypothèse des effets facilitateurs. Regel et al. [9, 42] n'ont pas trouvé d'effet facilitateur dans les tâches de TR simple ou impliquant un choix, et seulement des résultats variables dans les tâches de mémoire «n-back ». Keetley et al. [32] ont observé un déficit dans les tâches de TR simples et impliquant un choix. Cependant, ces résultats $(\mathrm{P}<0,005-0,043)$ n'ont pas été ajustés pour les tests multiples (18 tests) (voir également [43]). Curcio et al. [44] n'ont pas montré d'effet significatif dans les mêmes tâches de TR simple (le TR impliquant un choix n'ayant pas été testé).

Dans une tâche de soutien d'attention, Lee et al. [45] ne montrent pas d'effet après correction de la valeur de $\mathrm{P}$ en lien avec les comparaisons multiples (4 tests), malgré une tendance de réduction du TR $(\mathrm{P}<0,019)$. Eltiti et al. [46] ont rapporté l'absence d'effet significatif des signaux des stations de base GSM ou UMTS sur les fonctions d'attention ou de mémoire après l'application des corrections de Bonferroni (3 tests).

Besset et al. [47] et Fritzer et al. [48] n'ont pas montré d'effet d'expositions cumulatives à long terme de signaux GSM du téléphone sur les fonctions d'attention, de mémoire ou exécutives. Une méta-analyse conduite sur 10 études concernant les effets des signaux GSM sur les fonctions d'attention et de mémoire révélait une augmentation du niveau d'erreur dans le test «2-back nontarget » $(\Delta=0,05,95 \%$ CI: 0,03-0,07) [49]. En plus des fonctions d'attention et de mémoire, fréquemment étudiées, Maier et al. [50] ont rapporté une augmentation des seuils dans des tâches de mémoire auditive, mais d'autres études ont montré l'absence d'effet des signaux $2 \mathrm{G}$ sur les seuils visuels de discrimination de luminance [51], les seuils critiques de fusion d'images[52], le traitement de l'information visio-motrice [53] et les saccades [54]. Aucune des études sur les signaux 3G (ou UMTS) n'a montré d'effet significatif sur différentes fonctions cognitives [46, 55-58]. Dans ces études, quelques biais limitent l'interprétation des données. Eliyahu et al. [59] et Luria et al. [60] ont combiné les données du côté droit et du côté exposé sham pour obtenir des résultats significatifs par rapport au côté gauche exposé. Smythe et Costall [61] ont comparé seulement deux groupes avec exposition réelle ou sham parce que la comparaison initiale, comprenant une condition « sans téléphone », ne montrait pas de résultat.

En résumé, les premières études de comportement reportaient des effets significatifs de l'exposition RF du téléphone portable sur les fonctions d'attention et de mémoire (amélioration des performances cognitives), qui semblaient avoir eu lieu par hasard en raison de comparaisons multiples. Les études plus récentes tenant compte des comparaisons multiples, n'ont pas reproduit les résultats précédents. Dernièrement, les études sur les fonctions cognitives autres que l'attention et la mémoire ou sur les signaux 3G n'ont pas montré d'effet significatif.

\section{Effets sur la vascularisation}

Les effets les plus cohérents d'une exposition aiguë sur les sujets humains sont les réponses thermorégulatrices liées à l'échauffement induit par les RF, dont les plus immédiates sont la vasodilatation cutanée et l'évaporation liée la sudation.

Quelques modifications du flux sanguin cérébral régional (FSCr) ont été publiées pendant et après une exposition RF, mais les données disponibles sont équivoques [8, 35, 62-65]. Huber et al. [8] ont observé une augmentation significative de 5 à $7 \%$ du FSCr dans le cortex préfrontal dorsolatéral de l'hémisphère gauche du cerveau après 30 minutes d'exposition à un téléphone GSM. Le flux sanguin a été mesuré par tomographie par émission de positons (TEP) de l'oxygène. Quelques années plus tard, ils ont confirmé cet effet avec des RF modulées par impulsions (comme dans le signal du téléphone mobile), mais pas avec une émission continue comme le signal des antennes relais, au même DAS pic de $1 \mathrm{~W} . \mathrm{kg}^{-1}$ [64]. Haarala et al. [35] ont trouvé une diminution bilatérale du FSCr dans le cortex auditif avec une exposition pendant l'examen TEP : ils ont donc trouvé une diminution pendant l'examen, tandis qu'Huber et al. [8] ont trouvé une augmentation après l'examen. Quand Aalto et al. [63] ont amélioré le protocole précédent en éliminant de possibles artefacts acoustiques, ils ont également observé une diminution du FSCr près de l'antenne, et une augmentation dans d'autres régions variées plus profondes dans le cerveau. Dans leur revue, Kwon et Hamalainen [24] concluent que les résultats des mesures hémodynamiques de réponse cérébrale sont prometteurs mais encore peu nombreux et non concluants. Toutefois ces résultats, quoique non convergents, ne sont pas non plus contradictoires : ils 
indiquent une diminution de débit pendant l'exposition dans le cortex auditif à proximité des antennes émettrices et une augmentation plus tardive dans d'autres régions du cerveau plus éloignées qui pourrait être réactionnelle. D'autres travaux sont nécessaires pour établir si une telle cohérence spatiale et temporelle existe ou si ces résultats sont aléatoires et non cohérents.

En lien avec le flux sanguin cérébral, une autre étude plus récente par Volkow et al. [65] a montré par PET du glucose, une augmentation du métabolisme cérébral local après exposition à un téléphone portable en mode réception. Ce résultat va dans le sens de ceux de Huber et al. Cependant l'échauffement comparatif du téléphone allumé ou éteint n'était pas contrôlé ou pas mentionné. Il n'est alors pas évident de savoir si l'effet est réellement dû à l'émission RF ou à une réaction intra-crânienne indirecte à l'échauffement du visage par l'électronique du téléphone portable.

En résumé, l'exposition aux RF du téléphone portable peut induire une augmentation ou une diminution du flux sanguin régional, selon la technique utilisée et le moment ou la zone d'enregistrement. L'échauffement par conduction de chaleur des téléphones devrait être strictement contrôlé entre les dispositifs réels et fictifs.

Ces changements du FSCr ne constituent pas en soi une altération de la santé. Des modifications du FSCr pourraient refléter ou produire des modifications localisées de l'activité neurale.

Tableau 1. Flux sanguin cérébral régional

\begin{tabular}{|c|c|c|c|c|}
\hline Paramètres mesurés & Conditions d'exposition & Réponse & Commentaires & Références \\
\hline $\begin{array}{l}\text { FSCr chez des } \\
\text { sains, } 20-25 \text { ans }(n=13) \text {. } \\
\text { TEP } 10 \quad \text { min } \\
\text { exposition. }\end{array}$ & $\begin{array}{l}902 \mathrm{MHz} \text {, signal d'un téléphone } \\
\text { mobile, DAS }=1 \mathrm{~W} \mathrm{~kg}^{-1} \text {, pendant } 30 \\
\text { min, } 1 \text { semaine entre l'exposition } \\
\text { réelle et l'exposition fictive (témoin). }\end{array}$ & $\begin{array}{l}\text { FSCr relativement augmenté } \\
\text { dans le cortex dorsolateral } \\
\text { prefrontal du côté de } \\
\text { l'exposition. }\end{array}$ & $\begin{array}{l}\text { Exposition moins } \\
\text { localisée qu'avec } \\
\text { un téléphone } \\
\text { mobile réel. }\end{array}$ & $\begin{array}{l}\text { Huber et al, } \\
2002\end{array}$ \\
\hline $\begin{array}{l}\text { FSCr pendant une tâche de } \\
\text { mémorisation chez des } \\
\text { volontaires sains, 21-35 } \\
\text { ans }(n=14) \text {. TEP pendant } \\
\text { l'exposition. }\end{array}$ & $\begin{array}{l}\text { Téléphone mobile GSM à } 902 \mathrm{MHz} \text {, } \\
\text { fonctionnant à } 0,25 \mathrm{~W} \text { (DAS 1,2 W. } \\
\mathrm{kg}^{-1} \text { ), pendant } 45 \text { min ; pas } \\
\mathrm{d}^{\prime} \text { 'intervalle entre l'exposition réelle } \\
\text { et l'exposition fictive. }\end{array}$ & $\begin{array}{l}\text { Diminution relative du FSCr } \\
\text { dans le cortex auditif, pas dans } \\
\text { la zone d'exposition RF } \\
\text { maximale. }\end{array}$ & $\begin{array}{l}\text { Signal auditif } \\
\text { possible. Séquence } \\
\text { d'événements pas } \\
\text { claire. }\end{array}$ & $\begin{array}{l}\text { Haarala et al., } \\
\text { 2003a }\end{array}$ \\
\hline $\begin{array}{l}\text { FSCr chez des } \\
\text { sains, } 20-25 \text { ans } \\
\text { TEP } \quad 10 \text { min }=12) \text {. } \\
\text { exposition. }\end{array}$ & $\begin{array}{l}902 \mathrm{MHz} \text {, signal d'un téléphone } \\
\text { mobile ou d'une station de base, } \\
\text { DAS }=1 \mathrm{~W} \mathrm{~kg}{ }^{-1} \text {, pendant } 30 \mathrm{~min}, 1 \\
\text { semaine entre l'exposition réelle et } \\
\text { l'exposition fictive (témoin). }\end{array}$ & $\begin{array}{l}\text { FSCr relativement augmenté } \\
\text { dans le cortex dorsolateral } \\
\text { prefrontal du côté de } \\
\text { l'exposition, avec le signal d'un } \\
\text { téléphone mobile, pas avec celui } \\
\text { d'une station de base. Pas de } \\
\text { lien avec la distribution locale } \\
\text { du DAS. }\end{array}$ & $\begin{array}{l}\text { Exposition moins } \\
\text { localisée qu'avec } \\
\text { un téléphone } \\
\text { mobile réel. }\end{array}$ & $\begin{array}{l}\text { Huber et al, } \\
2005\end{array}$ \\
\hline $\begin{array}{l}\text { FSCr par TEP pendant une } \\
\text { tâche cognitive et pendant } \\
\text { l'exposition à un téléphone } \\
\text { mobile chez des hommes } \\
\text { sains, } 25 \text { ans } \pm 2(n=12)\end{array}$ & $\begin{array}{l}\text { Téléphone mobile GSM à } 902 \mathrm{MHz} \text {, } \\
\text { fonctionnant à } 0,25 \mathrm{~W} \text { pendant } 30 \\
\text { min. }\end{array}$ & $\begin{array}{l}\text { Diminution du FSCr près de } \\
\text { l'antenne, augmentation dans } \\
\text { d'autres zones diverses. Pas } \\
\text { d'effet sur le temps de réaction. }\end{array}$ & $\begin{array}{l}\text { Séquence } \\
\text { d'événements pas } \\
\text { claire. }\end{array}$ & $\begin{array}{l}\text { Aalto et al, } \\
2006\end{array}$ \\
\hline $\begin{array}{l}\text { Métabolisme du glucose } \\
\text { par } \\
\left({ }^{18} \text { F)fluorodeoxyglucose }\right. \\
\left({ }^{18} \text { FDG). }\right.\end{array}$ & $\begin{array}{l}\text { Téléphone mobile à } 837,8 \mathrm{MHz} \text {, } \\
\text { fonctionnant en mode réception } \\
\text { pendant } 50 \mathrm{~min} \text {. }\end{array}$ & $\begin{array}{l}\text { Augmentation du métabolisme } \\
\text { du glucose dans quelques zones } \\
\text { localisées : cortex orbitofrontal } \\
\text { droit et partie inférieure du } \\
\text { gyrus temporal supérieur droit. }\end{array}$ & $\begin{array}{l}\text { Pas de contrôle de } \\
\text { la température }\end{array}$ & $\begin{array}{l}\text { Volkow et al., } \\
2011\end{array}$ \\
\hline
\end{tabular}

Par ailleurs, il n'y a pas d'indication claire qu'une exposition RF du type téléphone portable ait un effet sur la fréquence cardiaque au repos ou sur la tension artérielle [66-70]. Cependant, de légères modifications ont été rapportées sur la variabilité de la fréquence cardiaque dans deux études contradictoires [71, 72].

\section{Conclusion}

L'ensemble des travaux analysés fait ressortir un effet reproductible sur la puissance spectrale des ondes alpha de l'EEG, avec une amplitude augmentée notamment pendant le sommeil, et peut-être sur la vascularisation cérébrale. Les faibles modifications de l'activité électrique du cerveau et possiblement de la vascularisation cérébrale n'ont pas en soi de signification fonctionnelle. En dépit d'un grand nombre d'études, et bien que l'utilisation d'une grande variété de techniques d'évaluation des fonctions cognitives rende difficile une comparaison directe des résultats de ces différentes études, aucun effet robuste n'a été trouvé sur les fonctions cognitives.

Un avantage des études de laboratoire avec des volontaires humains est que les résultats indiquent la réponse probable d'autres personnes exposées dans les conditions semblables; les inconvénients incluent la durée souvent courte de l'exposition, du petit nombre et d'une plus grande hétérogénéité de volontaires, en comparaison aux études animales. Une conséquence est la puissance souvent faible pour détecter un effet significatif. En outre, les sujets sont habituellement choisis en bonne santé et peu susceptibles de refléter la gamme des réponses rencontrées dans 
l'ensemble de la population. Par exemple, les personnes très jeunes et les personnes âgées, ou les personnes bénéficiant d'un traitement pharmaceutique, ont rarement fait l'objet d'études expérimentales. Néanmoins, dans ce contexte limité, les études avec des volontaires apportent une contribution utile à l'étude des effets physiologiques de l'exposition chez les personnes en bonne santé. Il apparaît aussi important maintenant d'étudier les effets des champs RF chez les enfants et les adolescents, étant donné la connaissance croissante d'une maturation continue du cerveau jusqu'à un stade avancé de l'adolescence, et quelques études récentes de type écologique ont été effectuées dans cet objectif sur des écoliers. Des études cliniques seraient aussi utiles chez l'enfant, mais nécessitent des précautions éthiques et une validation physiologique préalable de la méthodologie et des paramètres analysés. Une étude de cohorte a démarré en France pour étudier l'influence de diverses expositions environnementales sur des enfants suivis de la naissance jusqu'à l'âge adulte, la cohorte ELFE. Une quantification de l'exposition aux champs électromagnétiques dans cette cohorte est prévue.

\section{Références bibliographiques}

[1] C.M. Cook et al., Exposure to ELF magnetic and ELF-modulated radiofrequency fields: The time course of physiological and cognitive effects observed in recent studies (2001-2005). Bioelectromagnetics 27(8) (2006) 613-627.

[2] J. Röschke, K. Mann, No short-term effects of digital mobile radio telephone on the awake human electroencephalogram. Bioelectromagnetics 18 (1997) 172-176.

[3] M. Hietanen et al., Human brain activity during exposure to radiofrequency fields emitted by cellular phones. Scand. J. Work Environ. Health 26 (2000) 87-92.

[4] A.V. Kramarenko, and Tan, U., Effects of high-frequency electromagnetic fields on human EEG: A brain mapping study. . Int. J. Neurosci. 113 (2003) 1007-1019.

[5] H. Hinrikus et al., Changes in human EEG caused by low level modulated microwave stimulation. . Bioelectromagnetics 25 (2004) 431-440

[6] F. Vecchio et al., Mobile phone emission modulates interhemispheric functional coupling of EEG alpha rhythms. Eur. J. Neurosci. 25 (2007) 1908-1913.

[7] H. Reiser et al., The influence of electromagnetic fields on human brain activity. Eur. J. Med. Res. 1 (1995) $27-32$.

[8] R. Huber et al., Electromagnetic fields, such as those from mobile phones, alter regional cerebral blood flow and sleep and waking EEG. Journal of Sleep Research 11(4) (2002) 289-295.

[9] S. Regel et al., Pulsed radio frequency radiation affects cognitive performance and the waking electroencephalogram. . Neuroreport 18 (2007) 803-807.

[10] G. Curcio et al., Is the brain influenced by a phone call? An EEG study of resting wakefulness. . Neurosci. Res. 53 (2005) 265-270.

[11] C. Cook et al., Resting EEG is affected by exposure to a pulsed ELF magnetic field. Bioelectromagnetics 25(3) (2004) 196-203.

[12] H. Hinrikus et al., Effect of 7, 14 and $21 \mathrm{~Hz}$ modulated $450 \mathrm{MHz}$ microwave radiation on human electroencephalographic rhythms Int J Radiat. Biol. 84 (2008) 69-79.

[13] R. Croft et al., The effect of mobile phone electromagnetic fields on the alpha rhythm of human electroencephalogram. . Bioelectromagnetics (2008);29:1-10.

[14] R. Croft, Leung, S, McKenzie, RJ, Loughran, SP, Iskra, S, Hamblin, DL, Cooper, NR., Effects of 2G and 3G mobile phones on human alpha rhythms: Resting EEG in adolescents, young adults, and the elderly. . Bioelectromagnetics 31(6) (2010) 434-44.

[15] R. Huber et al., Exposure to pulsed high-frequency electromagnetic field during waking affects human sleep EEG. Neuroreport 11(15) (2000) 3321-3325.

[16] R.J. Croft et al., Acute mobile phone operation affects neural function in humans. . Clin. Neurophysiol. 113 (2002) $1623-1632$.

[17] P. Wagner et al., Human sleep under the influence of pulsed radiofrequency electromagnetic fields: A polysomnographic study using standardized conditions. Bioelectromagnetics 19(3) (1998) 199-202.

[18] K. Mann, J. Röschke, Effects of pulsed high-frequency electromagnetic fields on human sleep. . Neuropsychobiology 33 (1996) 41-47.

[19] A.A. Borbély et al., Pulsed high-frequency electromagnetic field affect human sleep and sleep electroencephalogram. . Neurosci. Lett. 275(20) (1999) 207-210.

[20] N. Lebedeva et al., Investigation of brain potentials in sleeping humans exposed to the electromagnetic field of mobile phones. Critical Reviews in Biomedical Engineering 29(1) (2001) 125-133.

[21] S.P. Loughran et al., The effect of electromagnetic fields emitted by mobile phones on human sleep. Neuroreport 16(17) (2005) 1973-1976.

[22] A.A. Borbély, Huber, R., Graf, T., Fuchs, B., Gallmann, E., and Achermann, P., Pulsed high-frequency electromagnetic field affect human sleep and sleep electroencephalogram. . Neurosci. Lett. 275(20) (1999) 207-210. 
[23] C.S. Hung et al., Mobile phone 'talk-mode' signal delays EEG-determined sleep onset. Neuroscience Letters 421(1) (2007) 82-86.

[24] M.S. Kwon, H. Hamalainen, Effects of Mobile Phone Electromagnetic Fields: Critical Evaluation of Behavioral and Neurophysiological Studies. Bioelectromagnetics 32(4) (2011) 253-272.

[25] A. Preece et al., Effect of a $915-\mathrm{MHz}$ simulated mobile phone signal on cognitive function in man. International Journal of Radiation Biology 75(4) (1999) 447-456.

[26] M. Koivisto et al., The effects of electromagnetic field emitted by GSM phones on working memory. Neuroreport 11(8) (2000) 1641-1643.

[27] M. Koivisto et al., Effects of $902 \mathrm{MHz}$ electromagnetic field emitted by cellular telephones on response times in humans. Neuroreport 11(2) (2000) 413-415.

[28] N. Edelstyn, A. Oldershaw, The acute effects of exposure to the electromagnetic field emitted by mobile phones on human attention. Neuroreport 13(1) (2002) 119-121.

[29] A. Preece et al., Effect of $902 \mathrm{MHz}$ mobile phone transmission on cognitive function in children. . Bioelectromagnetics 26 (Supp1 7) (2005) S138-S143.

[30] M. Koivisto et al., The effects of electromagnetic field emitted by GSM phones on working memory. Neuroreport 11(8) (2000a) 1641-1643.

[31] G. Curcio et al., Time-course of electromagnetic field effects on human performance and tympanic temperature. Neuroreport 15(1) (2004) 161-164.

[32] V. Keetley et al., Neuropsychological sequelae of digital mobile phone exposure in humans. Neuropsychologia 44(10) (2006) 1843-1848.

[33] C. Wiholm et al., Mobile phone exposure and spatial memory. Bioelectromagnetics 30 (2009) 59-65.

[34] C. Haarala et al., Effect of a $902 \mathrm{MHz}$ electromagnetic field emitted by mobile phones on human cognitive function: A replication study. Bioelectromagnetics 24 (2003) 283-288.

[35] C. Haarala et al., Effects of a $902 \mathrm{MHz}$ mobile phone on cerebral blood flow in humans: A PET study. Neuroreport 14 (2003) 2019-2023.

[36] C. Haarala et al., $902 \mathrm{MHz}$ mobile phone does not affect short term memory in humans. Bioelectromagnetics 25 (2004) 452-456.

[37] C. Haarala et al., Pulsed and continuous wave mobile phone exposure over left versus right hemisphere: Effects on human cognitive function. Bioelectromagnetics 28 (2007) 289-295.

[38] C. Haarala et al., Electromagnetic field emitted by $902 \mathrm{MHz}$ mobile phones shows no effects on children's cognitive function. Bioelectromagnetics 26 (Suppl 7) (2005) S144-S150.

[39] R. Russo, Fox, E, Cinel, C, Boldini, A, Defeyter, MA, Mirshekar-Syahkal, D, Mehta, A. , Does acute exposure to mobile phones affect human attention? :. Bioelectromagnetics 27 (2006) 215-220.

[40] C. Cinel et al., Does the use of mobile phones affect human short-term memory or attention? . Appl Cogn Psychol 22 (2008) 1113-1125.

[41] C. Cinel et al., Exposure to mobile phone electromagnetic fields and subjective symptoms: A double-blind study. . Psychosom Med 70 (2008) 345-348.

[42] S. Regel et al., Pulsed radio-frequency electromagnetic fields: Dose-dependent effects on sleep, the sleep EEG and cognitive performance. J Sleep Res 16 (2007) 253-258.

[43] M. Lewis, Mobile phones are good for you, p < 0.36! Observations on Keetley, Wood, Spong and Stough (2006). Neuropsychologia 45 (2007) 1580-1581.

[44] G. Curcio et al., Psychomotor performance is not influenced by brief repeated exposures to mobile phones. Bioelectromagnetics 29 (2008) 237-241.

[45] T. Lee et al., The effect of the duration of exposure to the electromagnetic field emitted by mobile phones on human attention. Neuroreport 14 (2003) 1361-1364.

[46] S. Eltiti et al., Short-term exposure to mobile phone base station signals does not affect cognitive functioning or physiological measures in individuals who report sensitivity to electromagnetic fields and controls. Bioelectromagnetics 30 (2009) 556-563.

[47] A. Besset et al., No effect on cognitive function from daily mobile phone use. Bioelectromagnetics 26(2) (2005) 102-108.

[48] G. Fritzer et al., Effects of short- and long-term pulsed radiofrequency electromagnetic fields on night sleep and cognitive functions in healthy subjects. Bioelectromagnetics 28(4) (2007) 316-325.

[49] A. Barth et al., A meta-analysis for neurobehavioural effects due to electromagnetic field exposure emitted by GSM mobile phones. Occupational and Environmental Medicine 65(5) (2008) 342-346.

[50] R. Maier et al., Effects of pulsed electromagnetic fields on cognitive processes-A pilot study on pulsed field interference with cognitive regeneration. Acta Neurol Scand 110 (2004) 46-52.

[51] L. Irlenbusch et al., Influence of a $902.4 \mathrm{MHz}$ GSM signal on the human visual system: Investigation of the discrimination

threshold. Bioelectromagnetics 28 (2007) 648-654.

[52] J. Wilen et al., Psychophysiological tests and provocation subjects with mobile phone related symptoms. Bioelectromagnetics 27(3) (2006) 204-214.

[53] Y. Terao et al., Effects of thirty-minute mobile phone use on visuo-motor reaction time. Clinical Neurophysiology 117(11) (2006) 2504-2511. 
[54] T. Terao et al., Effects of thirty-minute mobile phone exposure on saccades. Clin Neurophysiol : 118 (2007) 15451556.

[55] M. Unterlechner et al., No effect of an UMTS mobile phone-like electromagnetic field of $1.97 \mathrm{GHz}$ on human attention and reaction time. Bioelectromagnetics 29(2) (2008) 145-53.

[56] G. Schmid et al., No influence on selected parameters of human visual perception of $1970 \mathrm{MHz}$ UMTS-like exposure. Bioelectromagnetics 26(4) (2005) 243-250.

[57] S. Regel et al., UMTS base station-like exposure, well-being, and cognitive performance. Environ Health Perspect 114 (2006) 1270-1275.

[58] I. Riddervold et al., Cognitive function and symptoms in adults and adolescents in relation to RF radiation from UMTS base stations. Bioelectromagnetics 29 (2008) 257-267.

[59] I. Eliyahu et al., Effects of radiofrequency radiation emitted by cellular telephones on the cognitive functions of humans. Bioelectromagnetics 27 (2006) 119-126.

[60] R. Luria et al., Cognitive Effects of Radiation Emitted by Cellular Phones: The Influence of Exposure Side and Time. Bioelectromagnetics 30(3) (2009) 198-204.

[61] J. Smythe, B. Costall, Mobile phone use facilitates memory in male, but not female, subjects. Neuroreport 14 (2003) 243-246.

[62] M. Kwon, H. Hamalainen, Effects of Mobile Phone Electromagnetic Fields: Critical Evaluation of Behavioral and Neurophysiological Studies. Bioelectromagnetics 32(4) (2011) 253-272.

[63] S. Aalto et al., Mobile phone affects cerebral blood flow in humans. Journal of Cerebral Blood Flow and Metabolism 26(7) (2006) 885-890.

[64] R. Huber et al., Exposure to pulse pulsemodulated radio frequency electromagnetic fields affects regional cerebral blood flow. . Eur J Neurosci 21 (2005) 1000-1006.

[65] N.D. Volkow et al., Effects of Cell Phone Radiofrequency Signal Exposure on Brain Glucose Metabolism. JamaJournal of the American Medical Association 305(8) (2011) 808-813.

[66] A.T. Barker et al., The Effect of GSM and Tetra Mobile Handset Signals on Blood Pressure, Catechol Levels, and Heart Rate Variability. Bioelectromagnetics 28 (2007) 433-438.

[67] S. Braune et al., Resting Blood Pressure Increase during Exposure to a Radio-Frequency Electromagnetic Field. Lancet 351 (1998) 1857-1858.

[68] S. Braune et al., Influence of a radiofrequency electromagnetic field on cardiovascular and hormonal parameters of the autonomic nervous system in healthy individuals. Radiation Research 158(3) (2002) 352-356.

[69] K. Nam et al., Effects of RF Exposure of Teenagers and Adults by CDMA Cellular Phones. Bioelectromagnetics 27 (2006) 509-514.

[70] K. Tahvanainen et al., Effects of cellular phone use on ear canal temperature measured by NTC thermistors. Clin Physiol Funct Imaging 27 (2007) 162-172.

[71] R. Huber et al., Radio Frequency Electromagnetic Field Exposure In Humans: Estimation of Sar Distribution in The Brain, Effects On Sleep and Heart Rate. Bioelectromagnetics 24 (2003) 262-276.

[72] M. Parazzini et al., Electromagnetic Fields Produced by Gsm Cellular Phones and Heart Rate Variability. . Bioelectromagnetics 28 (2007) 122-129. 